\title{
High reliability of real-time visual data transmission using superposition coding with receiver diversity
}

\begin{abstract}
Supporting visual data applications in the real-time communication systems are among the most challenging issues over the next generation wireless communication systems. This challenge is further magnified by the fact that the quality of reception is highly sensitive to transmission delay, data losses and bit error rate (BER) in such applications. In this paper, we proposed Superposition Coding with Receiver Diversity (SPC-RD) scheme, which employs unequal error protection (UEP) to improve the error performance, maximize the received signal to noise ratio (SNR) and optimize the reliability of the transmission system. In the transmitter side, the visual data is divided into a number of different priority layers based on their effects on the reception quality. These layers are modulated individually where the highest priority layer is modulated with the highest UEP level against error-prone channels, and vice versa. These modulated signals are then superimposed together and transmitted via wireless Single-Input Multiple-Output (SIMO) Rayleigh fading channel. In the receiver side, three different diversity combining approaches; selection combining (SC), equal gain combining (EGC) and maximal ratio combining (MRC) are considered. The combined signal is then passed through a multiuser demodulator so-called the ordered successive interference cancellation (O-SIC) demodulator to reconstruct and separate the data layers. This demodulation technique is evaluated and compared with the traditionally maximum likelihood joint detection (MLJD) technique. Extensive simulations have been carried out to validate the various assertions. Under the assumption of equal transmission power, the simulation results illustrate that the proposed SPC-RD scheme provides a SNR gain of 14.5 $\mathrm{dB}$ over the Rayleigh fading channel at the diversity order of three for the acceptable BER level of 10-3 when BPSK scheme is exploited compared to the traditional equal error protection system. In addition, the proposed scheme with O-SIC demodulation technique achieves almost similar performance compared to MLJD technique but using less computational complexity.
\end{abstract}

Keyword: Real-time wireless data transmission; Receiver diversity; Superposition coding; UEP SIMO fading channels 